\title{
Sm16, a major component of Schistosoma mansoni cercarial excretory/secretory products, prevents macrophage classical activation and delays antigen processing
}

\author{
David E Sanin and Adrian P Mountford*
}

\begin{abstract}
Background: Schistosoma mansoni cercariae penetrate the skin by releasing excretory/secretory (E/S) products known as 0-3hRP, which are associated with immune modulation through Toll like receptor (TLR) signalling. Furthermore, these secretions contain Sm16, which when given to cells as a recombinant protein inhibits human monocyte derived cytokine responses to TLR4 and TLR3 ligands. Nonetheless, the extent and mechanism(s) of these inhibitory effects remain largely uncharacterized.

Methods: Murine bone marrow derived macrophages were exposed to different fractions of 0-3hRP, obtained via ultracentrifugation, or recombinant Sm16. These cells were exposed to the parasite molecules in combination with different TLR ligands, or Interferon gamma, and tested for the production of the cytokines IL-10 and IL-12p40, and their ability to process antigen.

Results: The immunomodulatory function of 0-3hRP is enriched predominantly in the pellet fraction, which contains a greater proportion of Sm16, also corroborating the ability of recombinant Sm16 to inhibit macrophage activation in response to TLR ligands. We further demonstrate that Sm16 blocks classical activation of macrophages to LPS or IFN- $\gamma$ stimulation in vitro, and that inhibition of macrophage classical activation is independent of TLR2 recognition. Finally we show that Sm16 shares the altered intracellular processing observed for 0-3hRP, and is able to delay antigen processing by macrophages.

Conclusions: Collectively, our findings show that Sm16 is a major component of S. mansoni cercarial E/S products, and is partly responsible for its immune-regulatory properties. Moreover, we propose that the mechanism employed by $\mathrm{Sm} 16$ to exert its inhibitory function is likely to be linked with alteration of endosomal trafficking and is not dependent on particular TLR receptors. Finally, we suggest that accumulation of Sm16 in the skin after percutaneous infection with S. mansoni cercariae could contribute to limiting dermal inflammation.
\end{abstract}

Keywords: Helminths, Macrophages, Toll like receptors, Schistosoma mansoni, Cercarial excretory/secretory products, Macrophage activation

\section{Background}

Schistosoma mansoni cercariae penetrate the skin by releasing excretory/secretory $(\mathrm{E} / \mathrm{S})$ products derived from the post and pre-acetabular glands of the parasite [1-3] which aid migration of larvae through the skin to reach blood vessels, thereby facilitating infection of the host

\footnotetext{
* Correspondence: adrian.mountford@york.ac.uk

Centre for Immunology and Infection, Department of Biology, University of York, York, UK
}

[4,5]. S. mansoni cercarial E/S products contain more than 50 different proteins $[1,6]$, and are largely released within the first three hours after transformation, hence this preparation has been termed $0-3$ hour released preparation $(0-3 \mathrm{hRP})$ [7]. These molecules are the first parasite-derived material encountered by innate immune cells (e.g. macrophages, neutrophils, and dendritic cells) in the skin and as such constitute the first line of defense against invading parasites. For example, macrophages in 
the skin take up secreted S. mansoni E/S products [3]. These secretions also induce a strong cytokine response from macrophages in a Toll like receptor (TLR) dependent manner [7] and are retained in early endosomes for longer than other immunogenic stimuli [3] suggesting that 03hRP conditions the way immune cells affect their environment and process phagocytized material [8].

The constituent(s) of 0-3hRP responsible for its potential immunomodulatory function are not known but several constituent molecules have theoretical roles [1,2]. Enzymes which allow remodeling of extracellular matrix necessary for parasite penetration of the skin are some of the most frequently identified proteins, of which the best studied is cercarial elastase with chymotrypsin activity enabling it to break skin elastin [5]. However, at least seven other elastases are secreted by larvae into the skin, alongside five metalloproteases, one of which is Invadolysin [2]. The only protein in 0-3hRP with a defined immunological function is Sm16 (Smp_113760), which is able to induce cell apoptosis if it reaches the cytosol [9], and effectively blocks signalling downstream of TLR4 and TLR3 in human monocytic cell lines [10]. Sm16 is expressed between the late developmental stage of the sporocyst in the intermediate molluscan host and the invading larvae, disappearing after $48 \mathrm{~h}$ of skin penetration [11]. Sm16 interference with cytokine responses by human monocytes is upstream of IRAK1 activation and NF- $\mathrm{kB}$ signalling [10], indicating that it's functions occur in close association with the earliest events of TLR signal transduction.

0 -3hRP is recognized by TLRs, whilst Sm16 is known to inhibit the function of these receptors $[7,10]$. Consequently, we investigated the distribution and function of Sm16 in different fractions of S. mansoni cercarial E/S products. The immunomodulatory function of $0-3 \mathrm{hRP}$ is enriched predominantly in its pellet fraction and we show that this fraction retains a greater proportion of Sm16 than the soluble fraction. We corroborate the ability of this protein to inhibit macrophage activation in response to TLR ligands, and further demonstrate that Sm16 is able to block classical activation of macrophages in vitro and that it functions independently of TLR2 recognition. Finally, we determine that $\mathrm{Sm} 16$ shares the altered intracellular processing as seen with $0-3 \mathrm{hRP}$, and has the potential to delay antigen processing. Collectively, our findings show that Sm16 is a major component of $S$. mansoni cercarial E/S products, with this protein being partly responsible for the regulatory function of these secretions.

\section{Methods}

Parasites and parasite-derived material

The life cycle of a Puerto Rican strain of Schistosoma mansoni (S. mansoni) was maintained in outbred NMR-I mice and Biomphalariaglabrata snails. Infective cercariae were obtained following exposure of snails with a patent infection to incandescent light for $2 \mathrm{~h}$ to induce the release of the parasites. Cercarial E/S products were produced as described previously [1,3,7]. Briefly, culture supernatants containing the $0-3$ hour released preparation (0-3hRP) were collected (ensuring whole larvae and parasite tails were discarded), and stored at $-20^{\circ} \mathrm{C}$ until required. Pooled supernatants were concentrated using filter spin columns with a molecular weight cut off of $3 \mathrm{kDa}$ (GE Life Sciences) and the protein content measured using the $\mathrm{BCA}^{\circ}$ protein assay (Thermo Scientific).

Recombinant Sm16 (rSm16), unlabelled or labelled with AlexaFluor ${ }^{\circ} 546$, was a gift from Dr Martin Gullberg, Umeå University, Sweden $[9,10]$.

\section{Fractionation of $0-3 \mathrm{hRP}$}

$0-3 \mathrm{hRP}$ was fractionated by centrifugation at $100,000 \mathrm{~g}$ for 1 hour at $4^{\circ} \mathrm{C}$ into a soluble preparation and a pellet. The soluble $0-3 \mathrm{hRP}$ preparation was denoted $0-3 \mathrm{hRP}$, whilst the pellet re-suspended using a vortex in an equivalent volume of PBS was denoted $0-3 h R P_{\mathrm{p}}$. The protein content of both preparations was quantified as specified above.

\section{SDS polyacrylamide gel electrophoresis (PAGE)}

$0-3 \mathrm{hRP}$ and its fractions were separated by SDS-PAGE under reducing conditions (1x NuPAGE ${ }^{\circ}$ Sample Reducing Agent; Life Technologies) on 4-12\% NuPAGE Bis-Tris Precast gels (Life Technologies) for $2 \mathrm{~h}$ at $200 \mathrm{~V}$ in $1 \mathrm{xNu}$ PAGE $^{\circ}$ MOPS SDS Running Buffer (Life Technologies). Gels were stained over-night using Brilliant Blue G concentrate (SIGMA), and imaged using a GelDoc ${ }^{\bullet}$ and ImageLab $^{\circ}$ by Biorad.

Selected protein bands were identified by tandem mass spectrometry (MS/MS) by the Proteomics division of the Bioscience Technology Facility (University of York, York, UK) using a Matrix assisted laser desorption ionization (MALDI)-MS and MS/MS are performed using a Bruker ultraflex III MALDI-Time of flight (TOF)/TOF.

\section{Western blot analysis}

0-3hRP fractions and rSm16 were transferred after SDSPAGE onto nitrocellulose membranes using an iBlot $^{\circ}$ Transfer Stack (Life Technologies). The membranes were then processed using the SnapID ${ }^{\circ}$ system (Millipore) blocked with PBS containing 1\% BSA, incubated first with rabbit anti-rSm16 antibody (1:5000) (gift from Dr Martin Gullberg, Umeå University, Sweden) for $10 \mathrm{~min}$, and then goat anti rabbit antibody (1:30000) conjugated to horseradish peroxidase (Abcam). SuperSignal ${ }^{\circ}$ West Pico chemiluminescence reagent (Thermo Scientific) was used to reveal labelled proteins using X-ray film imaging (GE Healthcare). 


\section{Animals}

All animals were bred and maintained in the Biological Services Facility at the Department of Biology, University of York, according to the standards laid out in the Animal's Scientific Procedures Act 1986, and housed in filtertopped cages under specific pathogen free conditions. The University of York Ethics committee approved all experimental work. Aged matched female C57BL/6 strain, or TLR2 deficient (TLR2 ${ }^{-/-}$) [12] mice between 6-10 weeks old were used for all experimental procedures.

\section{In vitro culture and stimulation of murine bone marrow- derived macrophages}

Bone marrow from both femurs and tibias was flushed with PBS using a $25 \mathrm{G}$ needle and the resulting cell suspensions filtered to remove bone and tissue debris. Aliquots of $5 \times 10^{6}$ cells were re-suspended in $\mathrm{DMEM}^{\bullet}$ medium (Gibco) containing 10\% FCS, 2 mM L-glutamine (Gibco), 1\% Pen/Strep (Gibco) and $50 \mu \mathrm{M}$ 2-mercaptoethanol (complete DMEM), supplemented with macrophage colony stimulating factor (M-CSF) obtained from culture supernatants of L929 murine fibroblast cell line. Bone marrow cell suspensions were cultured at $37^{\circ} \mathrm{C}$ and $5 \%$ $\mathrm{CO}_{2}$ in $10 \mathrm{~cm}$ culture dishes for 7 days prior to the collection of adherent cells which were re-suspended in complete DMEM and subsequently used as bone marrow derived macrophages (BMMФs). Stimulation assays were performed on $1 \times 10^{5} \mathrm{BMM} \phi \mathrm{s} /$ well (96 well plate) in $200 \mu \mathrm{l}$ of complete DMEM, containing different concentrations of parasite-derived material, or recombinant Sm16 (rSm16). BMMфs were stimulated in the same manner with $1 \mathrm{ng} / \mathrm{ml}$ lipopolysaccharide (LPS) (SIGMA-ALDRICH, from Escherichia coli 0111:B4), $25 \mu \mathrm{g} / \mathrm{ml}$ Polyinosinic:polycytidylic acid (Poly I:C) (SIGMA-ALDRICH) and $5 \mu \mathrm{g} / \mathrm{ml}$ Pam3CSK4 (InvivoGen).

\section{Flow cytometry}

BMMфs were incubated in round bottom 96 well plates with neat goat serum and $1 \mu \mathrm{g}$ anti CD16/CD32 monoclonal antibody (mAb) (eBiosciense), for $10 \mathrm{~min}$ at $4^{\circ} \mathrm{C}$ to prevent non-specific $\mathrm{mAb}$ binding to Fc-receptors. Cells were then labelled with mAb against F4/80 (BM8), CD11b (M1/70), CD11c (HL3) and MHC-II (M5/114) (all eBiosciences) in $10 \mu \mathrm{l}$ of $1 \%$ FCS in PBS (FACS buffer) for 30-45 min at $4^{\circ} \mathrm{C}$. Cells were washed and then subject to immediate acquisition by flow cytometry, or fixed in $100 \mu \mathrm{l} 2 \%$ paraformaldehyde (PFA) in PBS to enable acquisition at a later point. Antigen processing assays were carried out by exposing $\mathrm{rSm} 16$ treated or control BMM $\phi$ s to 100 AlexaFluor 488 conjugated $E$. coli BioParticles ${ }^{\oplus}$ (Life technologies) per cell for varying lengths of time. Cells were then fixed as described above and analysed by flow cytometry. All flow cytometry was acquired using the Cyan ADP analyser (DakoCytomation,
Stockport, UK). Data was analysed using FlowJo software v7.6.5 (Tree Star, Inc, Ashland, Oregon, USA).

\section{Enzyme linked immune absorbent assays}

Culture supernatants were collected from in vitro BMM $\phi \mathrm{s}$ cultures after 24 hours, as described above, for cytokine analysis. The amounts of IL-10 and IL-12p40 were determined using DuoSet ELISA kits (R\&D Systems).

\section{Griess assay}

The amount of nitrite as an indirect product of the production of NO, was measured using Griess Reagent kit (Life Technologies). Briefly, culture supernatants were incubated for $30 \mathrm{~min}$ with Griess reagent, alongside a standard curve supplied by the manufacturer. Absorbance was measured at $550 \mathrm{~nm}$, and concentrations estimated based on the standard curve.

\section{Confocal microscopy of BMMФs exposed to fluorescently labelled rSm16}

BMMФs were allowed to adhere to glass cover slips for two hours in 24 well plates $\left(1 \times 10^{6}\right.$ cells/well $)$ and then exposed to labelled $\mathrm{rSm} 16(50 \mu \mathrm{g} / \mathrm{ml})$ and/or Fluorescein isothiocyanate (FITC) conjugated DEXTRAN (DEXTRAN ${ }^{\text {FITC }}$ ) (SIGMA) for different periods of time. After washing, cells were fixed on to the cover slips for 20 min with 4\% PFA in PBS at room temperature. Cells were then incubated with DAPI $(2 \mu \mathrm{g} / \mathrm{ml})$ (SIGMA) for $5 \mathrm{~min}$, mounted onto a glass microscope slide using Prolong ${ }^{\bullet}$ Gold (Life technologies), sealed with nail varnish, and finally imaged.

Alternatively, cover slips were placed in $0.05 \%$ saponin $0.2 \%$ BSA (staining buffer) for $30 \mathrm{~min}$ at room temperature and then incubated for 1 hour with polyclonal rabbit antibody against Early endosome antigen (EEA)-1 (Abcam) (1:200). Cover slips were washed $3 \mathrm{x}$ and then probed for 1 hour with goat anti-rabbit Alexa Fluor 488 (Life technologies) (1:1000). Finally, cover slips were washed twice with DAPI $(2 \mu \mathrm{g} / \mathrm{ml})$ included in the second washing step. After rinsing with deionized water, cover slips were mounted on glass slides using Prolong ${ }^{\bullet}$ Gold as above.

All images were acquired using a Zeiss LSM 710 invert microscope using ZEN microscope software.

\section{Statistical analysis}

Analysis of Variance (ANOVA) and multiple comparisons tests (Two tailed T-test, Tukey's, Sidak's and Bonferroni's) were performed to establish statistically significant differences between the groups $\left({ }^{*}=\mathrm{p}<0.05,{ }^{* *}=\mathrm{p}<0.01 ;{ }^{* * * *}=\right.$ $\mathrm{p}<0.001, * * * * \mathrm{p}<0.0001)$ using the software package GraphPad Prism ${ }^{\oplus}$. Error bars represent the standard error of the mean (SEM), based on technical replicates. 


\section{Results}

Pellet fraction of S. mansoni E/S products induces IL-10 production

Macrophages derived from the bone marrow of mice are an abundant and widely used source of naïve cells for in vitro studies [13-16]. Consequently, BMM $\phi$ s defined on the basis of their expression of CD11b, F4/80, and MHC-II, but not CD11c (Additional file 1: Figure S1) were exposed overnight to $0-3 \mathrm{hRP}, 0-3 \mathrm{hRP}_{\mathrm{S}}, 0-3 \mathrm{hRP} \mathrm{P}_{\mathrm{B}}$ or left un-stimulated; $0-3 \mathrm{hRP}$ and $0-3 \mathrm{hRP}_{\mathrm{S}}$ were both used at $50 \mu \mathrm{g} / \mathrm{ml}$, whilst $0-3 \mathrm{hRP}_{\mathrm{P}}$ was used at a lower dose $(25 \mu \mathrm{g} / \mathrm{ml})$ due to limited availability of material. Nevertheless, $0-3 h R P_{P}$ induced 10 -fold more IL-10 than either 0$3 \mathrm{hRP}$, or $0-3 \mathrm{hRP}_{\mathrm{S}}$ (Figure $1 \mathrm{~A}$, both $\mathrm{p}<0.0001$ ). Conversely, IL-12p40 production was significantly lower in BMM $\phi$ s exposed to $0-3 h R P_{P}$ compared to $0-3 h R P(p<0.01)$, although there was no significant difference between IL$12 \mathrm{p} 40$ between $0-3 \mathrm{hRP}$ and $0-3 \mathrm{hRP}$ (Figure $1 \mathrm{~B}$ ).

As IL-10 induction is significantly increased in response to $0-3 \mathrm{hRP}_{\mathrm{P}}$, the capacity of this preparation to inhibit IL12p40 was examined. Therefore, BMMфs were exposed overnight to LPS $(1 \mathrm{ng} / \mathrm{ml})$ in the presence of increasing doses of $0-3 h R P_{P}$. Even low concentrations of $0-3 h R P_{P}$ $(2 \mu \mathrm{g} / \mathrm{ml})$ significantly reduced the amount of IL-12p40 produced by BMMфs exposed to $1 \mathrm{ng} / \mathrm{ml}$ LPS (Figure $1 \mathrm{C}$, $\mathrm{p}<0.0001$ ), and although IL-12p40 production was still higher than Media control at the highest dose of $0-3 h R P_{P}$ $(50 \mu \mathrm{g} / \mathrm{ml})$, this was expected, as $0-3 h R P_{\mathrm{P}}$ alone is able to induce significant quantities of IL-12p40 (Figure 1B). IL-10 production in the presence of LPS was enhanced in the presence of the greatest concentrations of $0-3 h R P_{P}$ (Figure $1 \mathrm{C}, \mathrm{p}<0.0001$ ). Notably, IL-12p40 production was significantly impaired at $2 \mu \mathrm{g} / \mathrm{ml}$ of $0-3 \mathrm{hRP}_{\mathrm{P}}(\mathrm{p}<0.0001$, Figure $1 \mathrm{C}$ ), whereas the production IL-10 at this dose was not significantly different compared to BMMфs stimulated with LPS only (Figure 1C). This observation suggests that inhibition of IL-12p40 is independent of IL-10 as IL-12p40 is significantly reduced even in the absence of IL-10. In line with this hypothesis, increasing doses of unfractionated $0-3 \mathrm{hRP}$ were unable to block IL-12p40 production by BMM $\phi$ s, stimulated with LPS, despite a significant increase in IL-10 production $(\mathrm{p}<0.0001$, Additional file 2: Figure S2).

\section{Sm16 is enriched in the pellet fraction of S. mansoni E/S products}

As the ability to induce IL-10 in BMM $\phi$ s differed between 0 -3hRPs and $0-3 h \mathrm{P}_{\mathrm{B}}$ the distribution of proteins in the two fractions was assessed by SDS-PAGE. $0-3 \mathrm{hRP}_{\mathrm{S}}$ retains approximately $75 \%$ of the protein content available in the
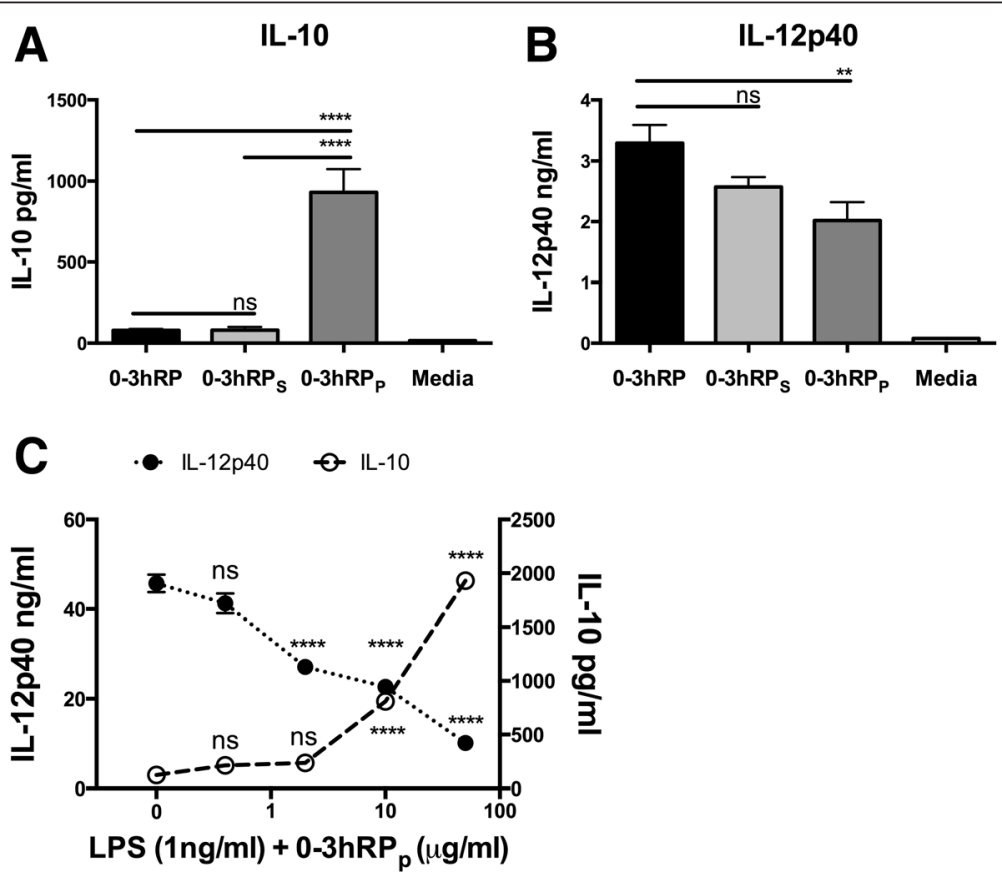

Figure 1 The pelleted fraction of cercarial E/S products $\left(0-3 h R P_{P}\right)$ induces abundant IL-10 from macrophages and blocks LPS driven IL-12p40. The presence of (A) IL-10 and (B) IL-12p40 in culture supernatants from BMMФs exposed to 0-3hRP $(50 \mu \mathrm{g} / \mathrm{ml}), 0-3 \mathrm{hRP}$ ( $(50 \mu \mathrm{gg} / \mathrm{ml})$, 0-3hRPp $(25 \mu \mathrm{g} / \mathrm{ml})$, or left un-stimulated (Media). (C) IL-12p40 (closed circles, left axis) and IL-10 (open circles, right axis) in culture supernatants of BMMФs exposed to LPS (1 ng/ml) and increasing doses of 0-3hRPp. Bars, or symbols, are mean values \pm SEM of 6 technical replicates and are representative of three independent experiments. ANOVA and Tukey's or Dunnett's test were performed to examine statistically significant differences between selected means $\left({ }^{* *}=p<0.01 ;{ }^{* * *}=p<0.0001 ; n s=p>0.05\right)$. 

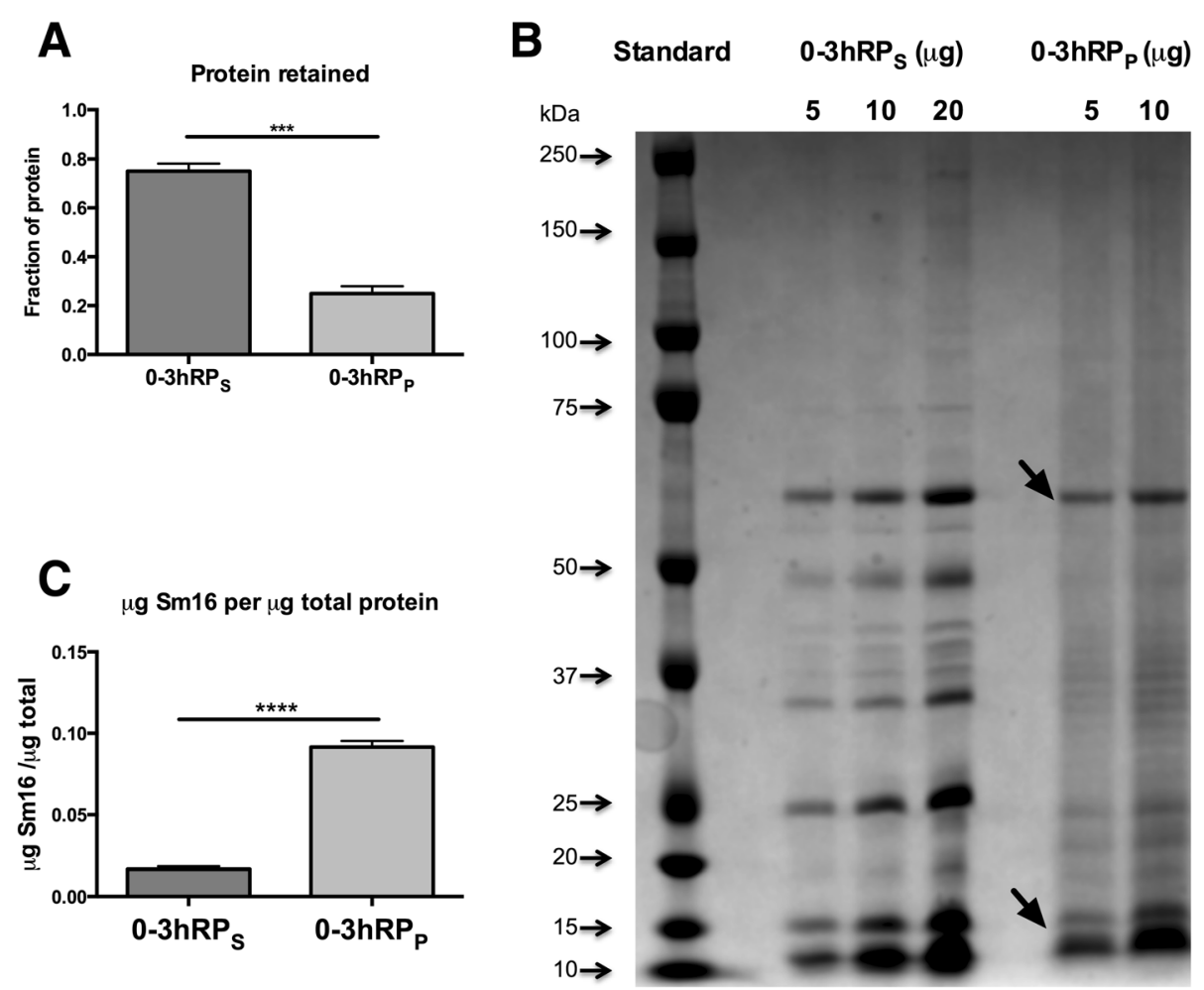

Figure $2 \mathrm{Sm} 16$ is enriched in pellet fraction of cercarial E/S products. Three preparations of 0-3hRP were fractionated by ultracentrifugation and the pellet fractions restored to the original volume of each preparation. (A) Protein content of each fraction expressed as a percentage of the total protein present in the original preparation. Bars = mean + SEM; statistical significance was tested using two tailed t-test $(* * *=p<0.001)$. (B) SDS PAGE gel of 0-3hRP fractions $(5,10$ and $20 \mu \mathrm{g}$ ) stained for protein. Black arrows highlight bands identified by mass spectrometry. (C) Equivalent volumes of $0-3 \mathrm{hRP}_{\mathrm{S}}(78 \mu \mathrm{g})$ and $0-3 \mathrm{hRP} \mathrm{P}(10 \mu \mathrm{g})$ based on the original preparation, were processed for Western blot analysis alongside $\mathrm{rSm} 16(1 \mu \mathrm{g})$ probed using rabbit anti-rSm16 antibody, estimated as relative concentration of Sm16 / $\mu \mathrm{g}$ 0-3hRP. Bars are mean + SEM, two tailedt-test show statistically significant differences $(* * * * p<0.0001)$.

original unfractionated 0 -3hRP preparation (Figure 2A), whereas the amount of protein in the $0-3 \mathrm{hRP}$ fraction was much lower ( 25\%). Analysis by SDS-PAGE revealed a number of discrete bands in the two fractions (Figure $2 \mathrm{~B}$ ) with $0-3 h R P_{P}$ being comprised of a much simpler range than $0-3 h R P_{S}$. Two of the most dominant bands in 0$3 \mathrm{hRP} \mathrm{P}_{\mathrm{P}}$ (Figure 2B, black arrows) were identified using MS/ MS, with the higher molecular weight band as Invadolysin (M08) (Smp_90100) with a mascot score of 826 and 7 peptides identified, whilst the lower molecular weight band contained Sm16 (Smp_113760), with a mascot score 70 and 2 peptides (Additional file 3: Table S1).

Western blot analysis of equivalent volumes of both fractions, where each fraction was reconstituted to the original starting volume of 0 -3hRP used to make the fractions, showed that anti-rSm16 antibody detected the native form of this protein in both $0-3 h R P_{S}$ and $0-3 h R P_{p}$ (Additional file 4: Figure S3), whilst densitometry analysis normalizing to pixel intensity of $\mathrm{rSm} 16$, indicates that $\mathrm{Sm} 16$ was enriched as a proportion in $0-3 \mathrm{hRP} P$ (greater than 5 fold) compared to $0-3 \mathrm{hRP} \mathrm{S}_{\mathrm{S}}$ (Figure $2 \mathrm{C}$, $\mathrm{p}<0.0001)$.
Recombinant Sm16 blocks BMMФs activation in response to TLR4 and TLR3, but not TLR2 ligands

BMM $\phi s$ exposed to LPS were unable to produce significant quantities of IL-12p40 and IL-10 when $\mathrm{rSm} 16$ $(10 \mu \mathrm{g} / \mathrm{ml})$ was present, whereas the Buffer control (phosphate buffer $\mathrm{pH} 7.5$, containing $0.45 \mathrm{M} \mathrm{NaCl}$ to prevent aggregation of $\mathrm{rSm} 16$ ) had no effect (Figure $3 \mathrm{~A} \& \mathrm{~B}$ ). Macrophage function is modulated in vivo by several cytokines, particularly IFN- $\gamma$, in the presence of ligands for TLR4. In this context, whilst IL-12p40 production to LPS in the presence of IFN- $\gamma(25 \mathrm{U} / \mathrm{ml})$ was enhanced, the addition of $\mathrm{rSm} 16$ significantly reduced IL-12p40 production (Figure $3 \mathrm{C}, \mathrm{p}<0.0001$ ). Furthermore, whilst a small amount of $\mathrm{NO}_{2}{ }^{-}$was produced by $\mathrm{BMM} \phi \mathrm{s}$ in response to LPS, this was reduced by the presence of $\mathrm{rSm} 16$ (Figure 3D, $\mathrm{p}<0.01$ ). Activation induced by IFN- $\gamma$, greatly enhanced the levels of $\mathrm{NO}_{2}^{-}$detected; however again $\mathrm{rSm} 16$ significantly reduced the levels of levels of $\mathrm{NO}_{2}^{-}$(Figure 3D, $\mathrm{p}<0.0001$ ).

With respect to ligands for other TLRs, BMM $\phi$ s stimulated with $25 \mu \mathrm{g} / \mathrm{ml}$ Poly I:C (ligand for TLR3) and rSm16 were also unable to produce IL-12p40 (Figure 4A) 


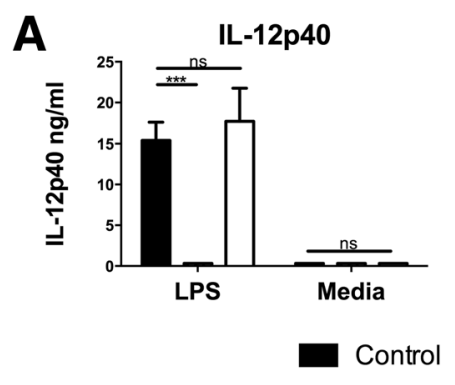

C

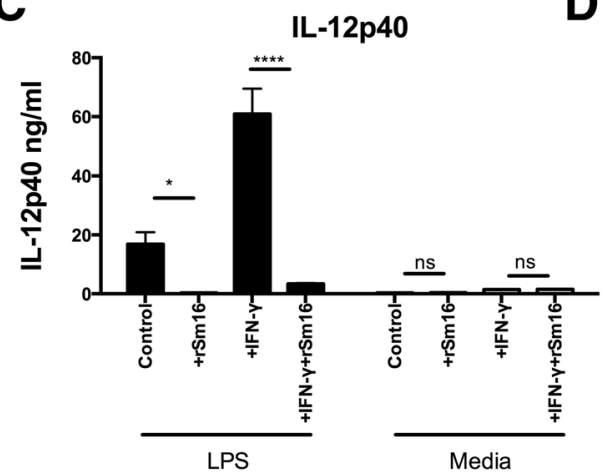

B

D
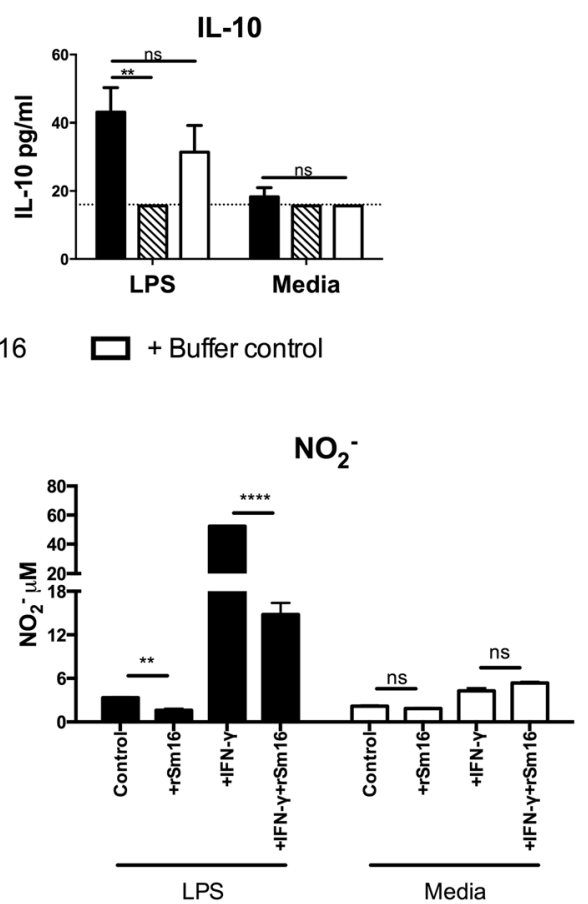

Figure 3 Recombinant Sm16 blocks activation of BMMФs in response to LPS and IFN-p. The presence of (A) IL-12p40 and (B) IL-10 in culture supernatants from BMMФs exposed to LPS (1 ng/ml) (black bars), or Media, in the presence of rSm16 (10 $\mu \mathrm{g} / \mathrm{ml})$ (hatched bars), or an equivalent volume of protein buffer (open bars). (C) IL-12p40 and (D) nitric oxide $\left(\mathrm{NO}_{2}^{-}\right)$in culture supernatants from BMMФs exposed to LPS $(1 \mathrm{ng} / \mathrm{ml})$ plus IFN- $\gamma(25 \mathrm{U} / \mathrm{ml})$ in the presence, or absence of $\mathrm{rSm} 16(10 \mu \mathrm{g} / \mathrm{ml})$. Bars = means + SEM of 3 technical replicates. Dotted line represents minimum level of cytokine detection by ELISA. Statistically significant differences tested by ANOVA and Bonferroni's or Sidak's test between selected means $\left(^{*}=p<0.05 ;{ }^{* *}=p<0.01 ;{ }^{* *}=p<0.001 ;{ }^{* * * *}=p<0.0001 ; n s=p>0.05\right)$. Results are representative of three independent experiments.

or IL-10 (Figure $4 \mathrm{C}$ ). However, the presence of rSm16 had no effect on cytokine production in BMMфs exposed to $5 \mu \mathrm{g} / \mathrm{ml}$ Pam3CSK4 (Figure $4 \mathrm{~B} \& \mathrm{D}$ ). Therefore, whilst $\mathrm{rSm} 16$ prevented TLR4 and TLR3 mediated activation of BMMфs, it appears to be unable to block signalling from TLR2.

Unlike LPS, Pam3CSK4 and Poly I:C, which are recognized by only one TLR, $S$. mansoni cercarial secretions require both TLR2 and TLR4 to induce cytokine production (Sanin et. al., manuscript in preparation). BMM $\phi \mathrm{s}$ stimulated with $50 \mu \mathrm{g} / \mathrm{ml} \mathrm{0-3hRP} \mathrm{alone} \mathrm{or} \mathrm{treated} \mathrm{with}$ rSm16, or buffer control, produced significantly less IL$12 \mathrm{p} 40$ ( $\mathrm{p}<0.01$, Additional file 5: Figure S4A) and significantly more IL-10 ( $<<0.001$, Additional file 5: Figure S4B). Thus, cytokine production by BMM $\phi$ s stimulated with $0-3 h R P$, supplemented with $\mathrm{rSm} 16$, is reminiscent of cytokine production by these cells exposed to $0-3 h R P_{P}$ (Figure 1A \& B). This is in line with our findings that $0-3 h R P_{P}$ has proportional more Sm16 than the unfractionated antigen. As BMM $\phi$ s require TLR2 to respond to 03hRP (Sanin et. al., manuscript in preparation), Sm16 too could assert its inhibitory function through this receptor. To confirm this, BMMфs from TLR2 ${ }^{-/-}$mice were stimulated with LPS, or Poly I:C, in the presence of rSm16. Both ligands induced robust IL-12p40 production in TLR2 ${ }^{-/-}$
BMMфs, but in both cases this was completely ablated by rSm16 (Figure 4E), demonstrating that rSm16 acts on BMM $\phi$ s independently of TLR2.

\section{Recombinant Sm16 is taken up by BMMФs using a distinct processing pathway}

BMМФs exposed to rSm16 labelled with AF594 $\left(\mathrm{rSm} 16^{\mathrm{AF594}}\right)$, revealed that $\mathrm{rSm} 16$ was closely associated with EEA-1 at $10 \mathrm{~min}$, and as late as $100 \mathrm{~min}$ after stimulation (Figure 5A). Indeed, EEA-1 appeared to surround $\mathrm{rSm} 16$ (Figure 5B, white arrows on inserts), staining which is frequently observed in immunofluorescence microscopy for EEA-1 [17], suggesting that $\mathrm{rSm} 16$ persists in early endosomes. This prolonged retention of $\mathrm{rSm} 16$ in early endosomes is reminiscent of previous observations for 0-3hRP [3].

The cellular fate of rSm16 in BMM $\phi s$ compared to readily processed DEXTRAN, as a marker of material being present in the phagolysosomes [18], demonstrated that 10 and 60 min after removal of these stimuli, both $\mathrm{rSm} 16^{\mathrm{AF594}}$ and DEXTRAN ${ }^{\mathrm{FITC}}$ were found in separate and discrete intracellular BMMфs compartments (Figure 5C). Whilst rSm16 ${ }^{\text {AF594 }}$ was abundant in the periphery of the cell, DEXTRAN $^{\text {FITC }}$ was closer to the nucleus. By 100 min, only a faint signal was detected for DEXTRAN ${ }^{\mathrm{FITC}}$. The 

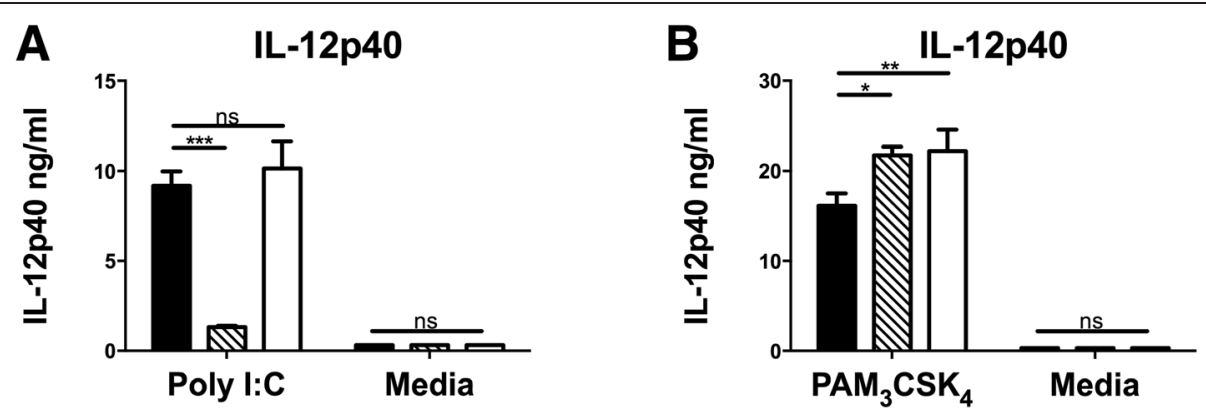

C

IL-10

D
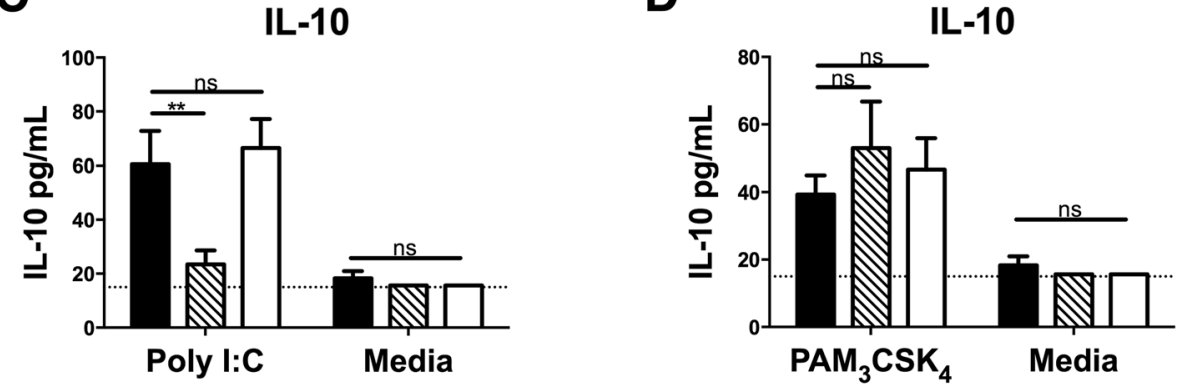

Control

$\mathbf{\nabla}+\mathrm{rSm} 16$

$\square+$ Buffer control

E

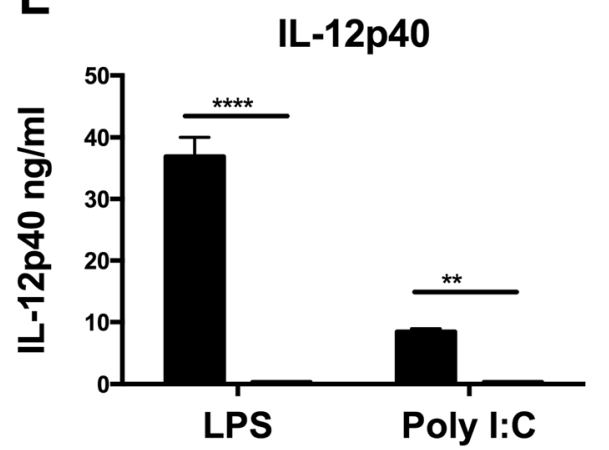

Figure 4 Recombinant Sm16 blocks cytokine production in BMMФs exposed to TLR4 and TLR3, but not TLR2 ligands. (A-B) IL-12p40

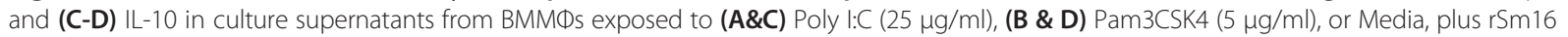
$(10 \mathrm{\mu g} / \mathrm{ml})$ (hatched bars) or an equivalent volume of protein buffer (open bars). Stimulus only controls (black bars) are also given. (E) BMMФs from

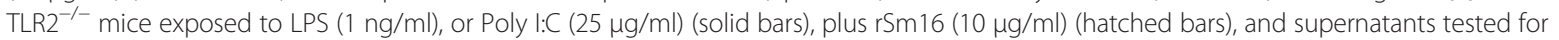
IL-12p40 by ELISA. Means + SEM of 3 technical replicates are presented. Dotted line represents minimum level of cytokine detection. ANOVA and Bonferroni's or Sidak's test were performed to examine statistically significant differences between the means $\left({ }^{*}=p<0.05 ;{ }^{* *}=p<0.01 ;{ }^{* * *}=\right.$ $\left.p<0.001 ;{ }^{* * *}=p<0.0001 ; n s=p>0.05\right)$. Results are representative of three independent experiments.

presence of $\mathrm{rSm} 16^{\mathrm{AF594}}$ was also greatly reduced, yet notably foci were still visible in the perinuclear region (Figure 5C, white arrows).

\section{Recombinant Sm16 treated BMMФs exhibit delayed antigen processing}

Prolonged retention of $\mathrm{rSm} 16$ in BMM $\phi$ s suggested that these cells might have a partial disruption of normal antigen processing. To address this question BMMфs were treated with $\mathrm{rSm} 16$ or left untreated (Media) and subsequently exposed to AlexaFluor ${ }^{\circ} 488$ conjugated $E$. coli BioParticles ${ }^{\oplus}$ (100 particles per cell) (Figure 6). The percentage of cells containing E. coli particles was determined to be significantly higher in $\mathrm{rSm} 16$ treated BMM $\phi \mathrm{s}$ compared to Media control after 30 and $100 \mathrm{~min}$ $(\mathrm{p}<0.0001$, Figure 6A \& B) post exposure. The median fluorescence intensity (MFI) of the population (representative overlaid histogram after $30 \mathrm{~min}$ given in Figure $6 \mathrm{C}$ ), as a measure of $E$. coli particles within BMMфs, was used to calculate the fold increase in the retention of Sm16 antigen, setting Media control arbitrarily to 1 (Figure 6D). In line with increased percentage of positive cells, BMM $\phi \mathrm{s}$ treated with $\mathrm{rSm} 16$ retained 2-fold more $E$. coli particles after $30 \mathrm{~min}(\mathrm{p}<0.0001)$ and this retention was still 


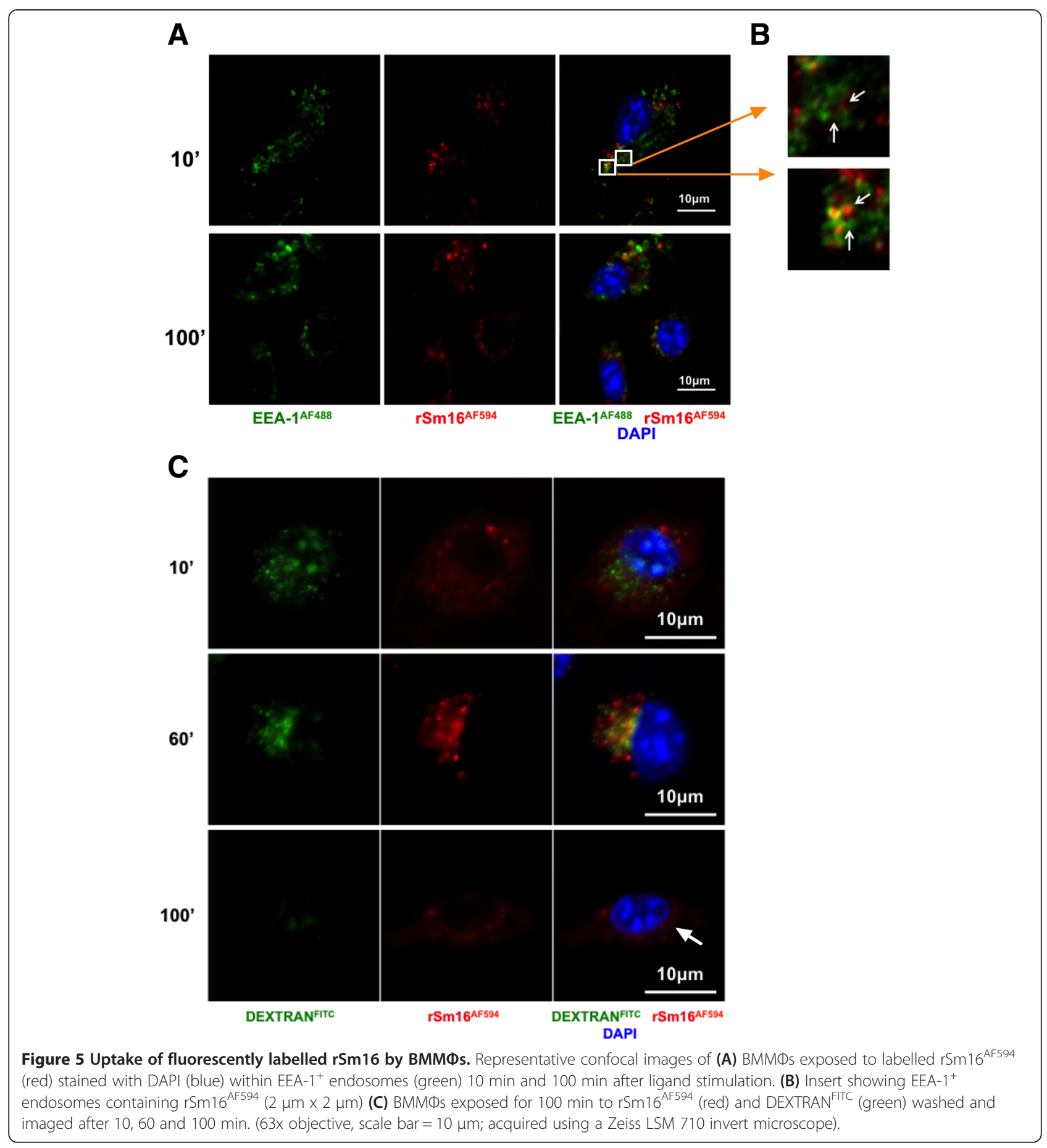

evident (albeit to a lesser degree) after $100 \mathrm{~min}$ (Figure 6D; $\mathrm{p}<0.01)$.

\section{Discussion}

In the present study we show that $\mathrm{Sm} 16$ is a major component of S. mansoni cercarial E/S products and as individuals living in areas endemic for schistosomiasis are liable to be frequently exposed to infective $S$. mansoni cercariae their E/S products, including Sm16, have the potential to provide a major stimulant of the innate immune system. Cercarial E/S products are composed of soluble and particulate fractions, with the latter most likely consisting of protein aggregates, which might accumulate in host tissues to a greater extent than the soluble fraction. As $0-3 h R P_{P}$ is produced as a pellet, its effect on BMMфs might only occur after proteolytic 

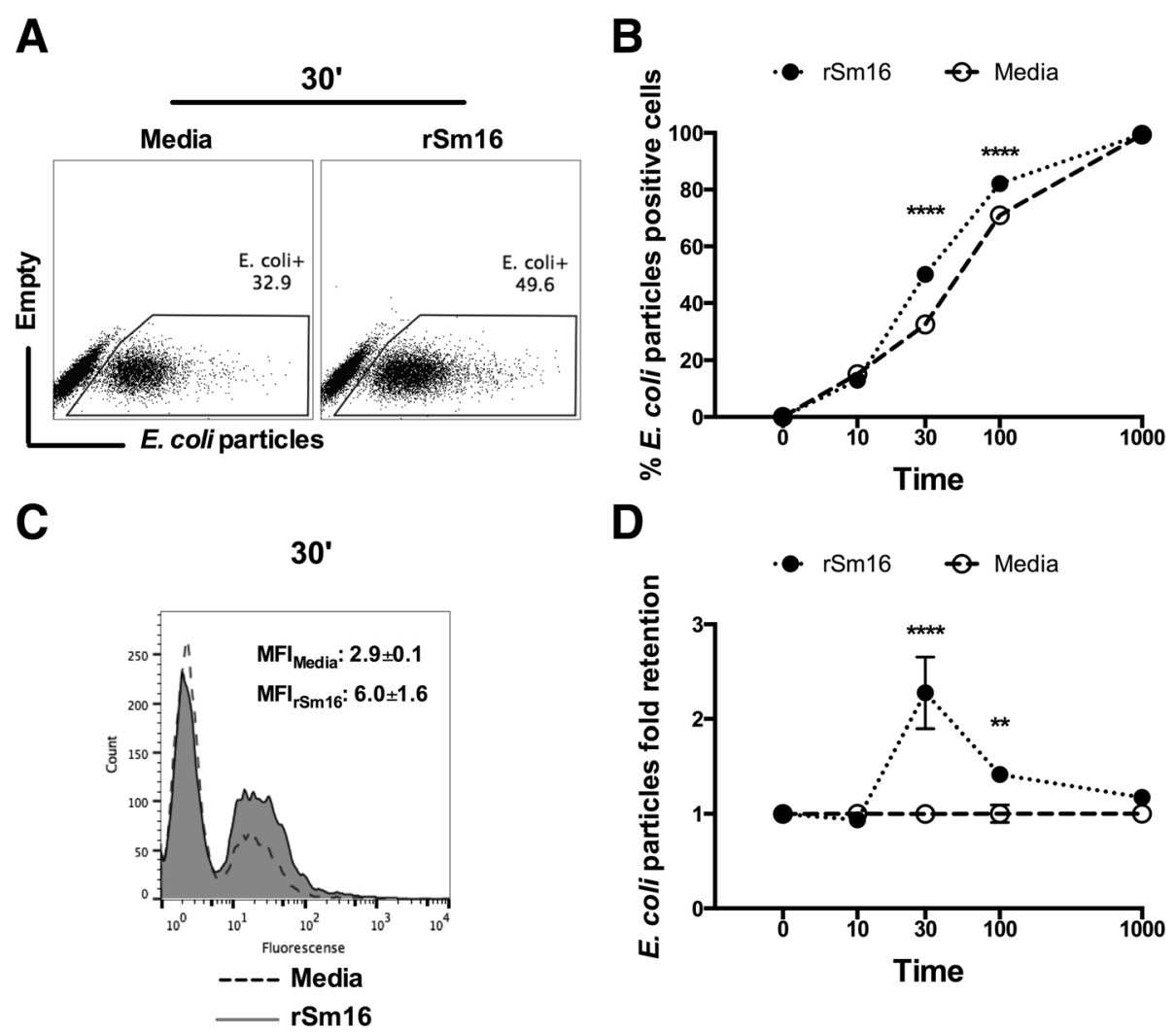

Figure 6 Recombinant Sm16 delays antigen processing by BMMФs. BMMФs were exposed for 10, 30, 100 or 1000 min to AlexaFluor ${ }^{\circledR} 488$ conjugated E. coli BioParticles ${ }^{\oplus}$ (100 particles per cell) in the absence (Media, open circles), or presence of rSm16 (10 $\mu \mathrm{g} / \mathrm{ml}$ ) (closed circles). (A) Representative flow plots of BMMФs 30 min after exposure to E. coli particles. (B) Mean percentages \pm SEM of BMMФs containing labelled E. coli BioParticles at each time point, and (C) representative overlaid histograms including the MFI \pm SD of each group. (D) Mean \pm SEM fold-retention of labelled E. coli BioParticles within rSm16-treated BMM $\varphi$ s, relative to the respective MFI of BMMФs treated with Media control, arbitrarily set to 1. Statistically significant differences between the means of $\mathrm{r} S \mathrm{~m} 16$ treated and Media controls at each time point were examined by ANOVA and Sidak's test $\left(^{* *}=p<0.01{ }^{* * * *}=p<0.0001\right)$.

degradation within these cells. Moreover, increased antiinflammatory activity observed in the pellet fraction of 0 -3hRP may be due to its higher Sm16 content ( 10\%).

We show that $\mathrm{rSm} 16$ inhibits cytokine production to TLR3 and TLR4 ligands in murine macrophages in a manner similar to previous observations using human cells where induction of IL-6 and IL-1RA was impaired [10]. Furthermore, we show that Sm16 also prevents IL12 p 40 production by macrophages that could restrict the induction of IL-12-driven Th1 cell responses [19-22]. Indeed, multiple exposures of skin to $S$. mansoni cercariae (and therefore greater quantities of E/S products, including Sm16) leads to lymphocytes in the skin draining lymph nodes becoming hypo-responsive in terms of Th cell associated cytokine production (Prendergast et. al., manuscript submitted) [23]. Infection with Sm16 deficient parasites (obtained through RNA silencing, or genetic manipulation) could ideally prove conclusively the role of this protein in the course of a skin infection, but these tools are not yet available [24].
In addition to its ability to inhibit innate immune cell responses to TLR ligands, Sm16 prevented classical activation of macrophages which ordinarily occurs in the presence of IFN- $\gamma$ leading to polarized Th1 responses [25]. Classically activated macrophages (CAM $\phi s)$ have increased bactericidal capacity as they have an elevated production of NO, and also produce elevated levels of IL-12, further supporting a Th1 environment [26]. However, Sm16 blocked IFN- $\gamma$ activation of macrophages in vitro, both by preventing IL-12p40 production and limiting NO production thereby restricting their ability to become classically activated. Consequently, Sm16 has the potential to impair both innate and adaptive immune responses in vivo in naturally infected hosts.

Immune modulation by helminth antigens has been traditionally linked to glycosylation, particularly since helminth E/S products are often heavily glycosylated [27-29]. Several proteins present in 0-3hRP are known to be heavily glycosylated [30], and glycans are partly responsible for the uptake of 0-3hRP [14]. However, Sm16 
is not glycosylated [30], and its immunomodulatory function is evident when expressed as a recombinant protein in eukaryotic cells from organisms other than S. mansoni, that have an inherently different glycosylation machinery $[9,10]$. Thus, we conclude that the regulatory function of Sm16 is independent of its glycosylation state.

Earlier reports on the inhibitory potential of Sm16 suggest that this occurs at very early stages of TLR signalling, as it can block both NF- $\mathrm{kB}$ activation and IRAK1 degradation [10]. Moreover, since 0-3hRP requires TLR2, TLR4 and MyD88 to induce cytokine production (Sanin et. al., manuscript in preparation) [7] and as its uptake is partly mediated by the mannose receptor [14], several other pattern recognition receptors are likely to be required to mediate the function of $0-3 \mathrm{hRP}$. However, direct competition with TLR ligands as a mechanism for Sm16 inhibition seems unlikely, as TLR ligands have diverse chemical structures. Furthermore, the fact that TLR4 and TLR3 use different adaptor molecules (MyD88 vs. TRIF) [31], but are both inhibited by Sm16, suggests that the inhibitory mechanism involving Sm16 is independent of either receptor. Moreover, TLR2 is able to scape the inhibitory effect of Sm16, further suggesting that adaptor molecules and downstream signalling pathways common between the three TLRs are unlikely to be the target of Sm16. Other helminth products inhibit TLR driven cytokine production by sequestration of downstream signalling machinery [32], and $0-3 \mathrm{hRP}$ is known to have altered endosomal processing [3]. If Sm16 exerts its function by limiting the availability of TLR signalling machinery, its effect would be then independent of TLR binding. To confirm this conclusion, we demonstrated that TLR2 signalling was indeed not required for the inhibitory action of Sm16. Thus, Sm16 inhibits TLR signalling using a mechanism that does not depend on direct binding to a TLR.

Sm16 was rapidly taken into macrophages and remained in early endosomes for a prolonged time (100 $\mathrm{min})$, reminiscent of 0-3hRP [3] and was not rapidly degraded. On the other hand, DEXTRAN, which is widely used as a lysosome tracker [33,34], was taken up within 10 minutes into a different intracellular compartment and then speedily degraded as it reached the lysosomes. In contrast, the intracellular fate of Sm16 had much slower degradation rates, further confirming our earlier observation of retention in early endosomes. Sm16 was also able to increase the retention of E. coli particles in macrophages, suggesting that overall antigen processing in these cells might be altered by $\mathrm{Sm} 16$. Phagosome maturation is influenced by TLR4 signalling [35], which Sm16 is able to block. Thus, the observed delayed antigen processing evidenced in this report could be as a result of Sm16 mediated TLR inhibition. However, as ligation of TLR4 can direct signalling from phagosomes [36,37], this opens up the possibility that $\mathrm{Sm} 16$ might be blocking TLR signalling by limiting antigen trafficking to these organelles.

\section{Conclusions}

We conclude that the particulate/pellet fraction of $S$. mansoni cercarial E/S material contains a significantly greater proportion of Sm16 than the soluble fraction, which may explain why the pellet fraction has a greater propensity to induce regulatory IL-10. The inhibitory activity of Sm16 operates upon TLR4 and TLR3 induced signalling but is not mediated by direct interaction with TLR2. Moreover, Sm16 is able to prevent classical activation of macrophages in the context of IFN- $\gamma$ stimulation. The mechanism employed by Sm16 to inhibit macrophage activation is likely to be linked with the rapid uptake and retention of this protein, which has a membrane binding properties [9], leading it to be quickly internalized. However, the rapid uptake of Sm16 does not lead to fast degradation, or indeed lysosomal trafficking, but instead results in retention in early endosomes leading to delayed processing. As with other helminth products, retention within intracellular components may possibly be responsible for mediating sequestration of essential TLR signalling machinery that subsequently blocks stimulation of specific TLRs [32]. Moreover, it appears that Sm16 extends this processing "defect" to E. coli particles, which exhibit enhanced retention in macrophages exposed to this protein, which could suggest that Sm16 prevents TLR signalling from within phagosomes. This could help explain why repeated exposure to $S$. mansoni cercariae, consequently leading to greater exposure to particulate fraction of cercarial secretions containing Sm16, leads to the inhibition of APC function and T cell responses in the course of infection [23].

\section{Additional files}

Additional file 1: Figure S1. Characterization of bone marrow cells as macrophages. Bone marrow cells cultured for 7 days were stained with (A) a viability dye and (B) labelled with antibodies against F4/80, CD11b, MHC-II and CD11c or relevant antibody isotype controls. Representative flow plots with percentages for numbers of cells within each gate from 7 independent experiments.

Additional file 2: Figure S2. Unfractionated cercarial E/S products (0-3hRP) do not block LPS driven production of IL-12p40. The presence of IL-12p40 (closed circles, left axis) and IL-10 (open circles, right axis) in culture supernatants of BMMФs exposed to LPS $(1 \mathrm{ng} / \mathrm{ml})$ and increasing doses of $0-3 h R P$. Symbols are mean values \pm SEM of 3 technical replicates and are representative of two independent experiments. ANOVA and Dunnett's test were performed to examine statistically significant differences between mean of LPS only control and LPS+0-3hRP treated BMMФs at each dose $\left({ }^{* * *}=p<0.0001 ; n s=p>0.05\right)$.

Additional file 3: Table S1.

Additional file 4: Figure S3. Western blot for $\mathrm{Sm} 16$ in fractions of cercarial E/S products. Equivalent volumes of $0-3 \mathrm{hRP} P_{\mathrm{S}}(78 \mu \mathrm{g})$ and $0-3 \mathrm{hRP}_{\mathrm{p}}(10 \mu \mathrm{g})$ based on the original preparation, plus an extra lane with 
2x 0-3hRP $(20 \mu \mathrm{g})$, were processed for Western blot analysis alongside rSm16 $(1 \mu \mathrm{g})$ and probed using rabbit anti-rSm16 antibody.

Additional file 5: Figure S4. BMMФs stimulated with recombinant Sm16 and S. mansoni cercarial secretions (0-3hRP) produced enhanced IL-10 and reduced IL-12p40. The presence of (A) IL-12p40 and (B) IL-10 in culture supernatants from BMMФs exposed to 0-3hRP $(50 \mu \mathrm{g} / \mathrm{ml})(\mathrm{black}$ bars), or Media, in the presence of $\mathrm{rSm} 16(10 \mu \mathrm{g} / \mathrm{ml})$ (hatched bars), or an equivalent volume of protein buffer (open bars). Bars are means +SEM of 3 technical replicates. Dotted line represents minimum level of cytokine detection. Statistically significant differences tested by ANOVA and Sidak's test between selected means ${ }^{* *}=p<0.01 ;{ }^{* *}=p<0.001 ;{ }^{* * *}=$ $p<0.0001 ; n s=p>0.05$ ). Results are representative of three independent experiments

\section{Competing interests}

The authors declare that they have no competing interests.

\section{Authors' contributions}

DES designed, performed and analysed the experiments; DES and APM wrote the paper. All authors read and approved the final manuscript.

\section{Acknowledgements}

The research was funded by The Wellcome Trust; project grant \# 092745/Z/ 10/Z to APM. DES was funded by the Fundación para el futuro de Colombia (COLFUTURO) and the Departamento Administrativo de Ciencia, Tecnología e Innovación de la República de Colombia (COLCIENCIAS).

The authors would like to thank Dr Martin Gullberg, Umeå University, Sweden for the generous gift of recombinant Sm16 and antibodies against this protein. We would also like to thank Dr Claire Bourke and Dr Catriona Prendergast for technical and scientific advice as well as the technical help given by Ann Bamford to maintain the schistosome life cycle and providing infective material. Finally, we thank Karen Hodgkinson and Graeme Park for help with flow cytometry and confocal microscopy, and Jerry Thomas and Adam Dowle for help with protein identification (Technology Facility, Department of Biology, University of York).

Received: 18 November 2014 Accepted: 16 December 2014 Published online: 06 January 2015

\section{References}

1. Curwen RS, Ashton PD, Sundaralingam S, Wilson RA: Identification of novel proteases and immunomodulators in the secretions of schistosome cercariae that facilitate host entry. Mol Cell Proteomics 2006, 5:835-844.

2. Wilson RA: Virulence factors of schistosomes. Microbes Infect 2012, 14:1442-1450.

3. Paveley RA, Aynsley SA, Cook PC, Turner JD, Mountford AP: Fluorescent imaging of antigen released by a skin-invading helminth reveals differential uptake and activation profiles by antigen presenting cells. PLoS Negl Trop Dis 2009, 3:e528.

4. McKerrow JH, Salter J: Invasion of skin by Schistosoma cercariae. Trends Parasitol 2002, 18:193-195.

5. Salter JP, Lim KC, Hansell E, Hsieh I, McKerrow JH: Schistosome invasion of human skin and degradation of dermal elastin are mediated by a single serine protease. J Biol Chem 2000, 275:38667-38673.

6. Knudsen GM, Medzihradszky KF, Lim KC, Hansell E, McKerrow JH: Proteomic analysis of Schistosoma mansoni cercarial secretions. Mol Cell Proteomics 2005, 4:1862-1875

7. Jenkins SJ, Hewitson JP, Ferret-Bernard S, Mountford AP: Schistosome larvae stimulate macrophage cytokine production through TLR4dependent and -independent pathways. Int Immunol 2005, 17:1409-1418.

8. Jenkins SJ, Hewitson JP, Jenkins GR, Mountford AP: Modulation of the host's immune response by schistosome larvae. Parasite Immunol 2005, 27:385-393

9. Holmfeldt P, Brannstrom K, Sellin ME, Segerman B, Carlsson SR, Gullberg M: The Schistosoma mansoni protein Sm16/SmSLP/SmSPO-1 is a membrane-binding protein that lacks the proposed microtubuleregulatory activity. Mol Biochem Parasitol 2007, 156:225-234.

10. Brännström $K$, Sellin $M E$, Holmfeldt $P$, Brattsand $M$, Gullberg $M$ : The Schistosoma mansoni protein Sm16/SmSLP/SmSPO-1 assembles into a nine-subunit oligomer with potential To inhibit Toll-like receptor signaling. Infect Immun 2009, 77:1144-1154.

11. Valle C, Festucci A, Calogero A, Macri P, Mecozzi B, Liberti P, Cioli D: Stage-specific expression of a Schistosoma mansoni polypeptide similar to the vertebrate regulatory protein stathmin. J Biol Chem 1999, 274:33869-33874.

12. Takeuchi O, Hoshino K, Kawai T, Sanjo H, Takada H, Ogawa T, Takeda K, Akira S: Differential roles of TLR2 and TLR4 in recognition of gram-negative and gram-positive bacterial cell wall components. Immunity 1999, 11:443-451.

13. Bailey MJ, Lacey DC, de Kok BV, Veith PD, Reynolds EC, Hamilton JA: Extracellular proteomes of M-CSF (CSF-1) and GM-CSF-dependent macrophages. Immunol Cell Biol 2011, 89:283-293.

14. Paveley RA, Aynsley SA, Turner JD, Bourke CD, Jenkins SJ, Cook PC, Martinez-Pomares L, Mountford AP: The Mannose Receptor (CD206) is an important pattern recognition receptor (PRR) in the detection of the infective stage of the helminth Schistosoma mansoni and modulates IFNgamma production. Int J Parasitol 2011, 41:1335-1345.

15. Medina EA, Morris IR, Berton MT: Phosphatidylinositol 3-Kinase Activation Attenuates the TLR2-Mediated Macrophage Proinflammatory Cytokine Response to Francisella tularensis Live Vaccine Strain. J Immunol 2010, 185:7562-7572

16. Saraiva M, Christensen JR, Tsytsykova AV, Goldfeld AE, Ley SC, Kioussis D, $\mathrm{O}$ Garra A: Identification of a macrophage-specific chromatin signature in the IL-10 locus. J Immunol 2005, 175:1041-1046.

17. Beas AO, Taupin V, Teodorof C, Nguyen LT, Garcia-Marcos M, Farquhar MG: Galphas promotes EEA1 endosome maturation and shuts down proliferative signaling through interaction with GIV (Girdin). Mol Biol Cell 2012, 23:4623-4634.

18. Humphries WH, Szymanski CJ, Payne CK: Endo-lysosomal vesicles positive for Rab7 and LAMP1 are terminal vesicles for the transport of dextran. PLoS One 2011, 6:e26626

19. de Jong EC, Vieira PL, Kalinski P, Schuitemaker JH, Tanaka Y, Wierenga EA, Yazdanbakhsh M, Kapsenberg ML: Microbial compounds selectively induce Th1 cell-promoting or Th2 cell-promoting dendritic cells in vitro with diverse th cell-polarizing signals. J Immunol 2002, 168:1704-1709.

20. Agrawal S, Agrawal A, Doughty B, Gerwitz A, Blenis J, Van Dyke T, Pulendran B: Cutting edge: different Toll-like receptor agonists instruct dendritic cells to induce distinct Th responses via differential modulation of extracellular signal-regulated kinase-mitogen-activated protein kinase and c-Fos. J Immunol 2003, 171:4984-4989.

21. Hogg KG, Kumkate S, Anderson S, Mountford AP: Interleukin-12 p40 secretion by cutaneous CD11c + and F4/80+ cells is a major feature of the innate immune response in mice that develop Th1-mediated protective immunity to Schistosoma mansoni. Infect Immun 2003, 71:3563-3571.

22. Yang $Z$, Zhang $X$, Darrah P, Mosser D: The regulation of Th1 responses by the p38 MAPK. I Immunol 2010, 185:6205-6213.

23. Cook PC, Aynsley SA, Turner JD, Jenkins GR, Van Rooijen N, Leeto M, Brombacher F, Mountford AP: Multiple helminth infection of the skin causes lymphocyte hypo-responsiveness mediated by Th2 conditioning of dermal myeloid cells. PLoS Pathog 2011, 7:e1001323.

24. Dvorak J, Beckmann S, Lim KC, Engel JC, Grevelding CG, McKerrow JH, Caffrey CR: Biolistic transformation of Schistosoma mansoni: Studies with modified reporter-gene constructs containing regulatory regions of protease genes. Mol Biochem Parasitol 2010, 170:37-40.

25. Barron L, Wynn TA: Macrophage activation governs schistosomiasisinduced inflammation and fibrosis. Eur J Immunol 2011, 41:2509-2514.

26. Benoit M, Desnues B, Mege JL: Macrophage polarization in bacterial infections. J Immunol 2008, 181:3733-3739.

27. Hokke CH, Deelder AM: Schistosome glycoconjugates in host-parasite interplay. Glycoconj J 2001, 18:573-587.

28. Hokke $\mathrm{CH}$, Yazdanbakhsh M: Schistosome glycans and innate immunity. Parasite Immunol 2005, 27:257-264.

29. Tundup S, Srivastava L, Harn DA Jr: Polarization of host immune responses by helminth-expressed glycans. Ann N Y Acad Sci 2012, 1253:E1-E13.

30. Jang-Lee J, Curwen RS, Ashton PD, Tissot B, Mathieson W, Panico M, Dell A, Wilson RA, Haslam SM: Glycomics analysis of Schistosoma mansoni egg and cercarial secretions. Mol Cell Proteomics 2007, 6:1485-1499.

31. Akira S, Takeda K: Toll-like receptor signalling. Nat Rev Immunol 2004, 4:499-511.

32. Pineda MA, Lumb F, Harnett MM, Harnett W: ES-62, a therapeutic antiinflammatory agent evolved by the filarial nematode Acanthocheilonema viteae. Mol Biochem Parasitol 2014, 194:1-8. 
33. Schmerk CL, Valvano MA: Burkholderia multivorans survival and trafficking within macrophages. J Med Microbiol 2013, 62:173-184.

34. Johnston SA, May RC: The human fungal pathogen Cryptococcus neoformans escapes macrophages by a phagosome emptying mechanism that is inhibited by Arp2/3 complex-mediated actin polymerisation. PLOS Pathog 2010, 6:e1001041.

35. Blander JM, Medzhitov R: Regulation of phagosome maturation by signals from toll-like receptors. Science 2004, 304:1014-1018.

36. Kagan JC, Iwasaki A: Phagosome as the organelle linking innate and adaptive immunity. Traffic 2012, 13:1053-1061.

37. Bonham KS, Orzalli MH, Hayashi K, Wolf Al, Glanemann C, Weninger W, Iwasaki A, Knipe DM, Kagan JC: A promiscuous lipid-binding protein diversifies the subcellular sites of toll-like receptor signal transduction. Cell 2014, 156:705-716.

\section{Submit your next manuscript to BioMed Central and take full advantage of:}

- Convenient online submission

- Thorough peer review

- No space constraints or color figure charges

- Immediate publication on acceptance

- Inclusion in PubMed, CAS, Scopus and Google Scholar

- Research which is freely available for redistribution 